\title{
Effects of Yogurt Supplementation and Exercise on Body Composition during Lactation
}

\section{Alexis Sharkey ${ }^{1}$, Lauren San Diego ${ }^{1}$, Tiffany Fuller ${ }^{2}$, Roberta Claro da Silva $^{1}$, Salam A Ibrahim ${ }^{1}$ and Heather L Colleran ${ }^{1 *}$ \\ ${ }^{1}$ Food and Nutritional Sciences, Department of Family and Consumer Sciences, North Carolina A\&T State University, United States \\ ${ }^{2}$ Department of Kinesiology, North Carolina A\&T State University, United States}

*Corresponding Author: Heather L Colleran, Food and Nutritional Sciences, Department of Family and Consumer Sciences, North Carolina A\&T State

University, United States.
Received: August 20, 2020

Published: September 11, 2020

(C) All rights are reserved by Heather $\mathbf{L}$

Colleran., et al.

\begin{abstract}
Breastfeeding reduces long-term maternal pregnancy weight retention but also results in high bone turnover. Dairy intake and exercise have bone-protective effects in pre-menopausal women during weight loss. The objective of this study was to investigate the effects of yogurt supplementation and exercise on body composition and bone mineral density in lactating women 8 - 20 weeks postpartum. At 8-wks postpartum, women were randomized into an intervention group [IG, $\mathrm{n}=6$ that received yogurt supplementation ( $\geq 3 \mathrm{~d} / \mathrm{wk}, 6 \mathrm{oz}$ each day) and exercise ( $3 \mathrm{~d} / \mathrm{wk}, 45 \mathrm{~min} / \mathrm{d}, \geq 10,000 \mathrm{steps} / \mathrm{d}$ )] or a control group (CG, $\mathrm{n}=2$, no dietary or exercise intervention) for 12-wks. Dual x-ray absorptiometry measured body composition (weight, fat mass, lean body mass) and bone mineral density. A submaximal treadmill test predicted VO2 max. Three-day dietary records were collected using Nutrition Data System for Research software. Due to COVID-19, results are reported without comparisons between groups. IG body composition and bone changes were similar to previous studies. Dietary intake did not differ between groups and both groups met the Recommended Dietary Allowance for carbohydrates and protein (g) intake. IG compliance for $3 \mathrm{~d} /$ wk of yogurt consumption and exercise was $97 \%$ and $90 \%$, respectively. The study provided preliminary data on dairy intake and exercise on body composition during lactation.
\end{abstract}

Keywords: Bone Mineral Density; Lactation; Exercise; Postpartum; Dairy

\section{Abbreviations}

AI: Adequate Intake; BMC: Bone Mineral Content; BMD: Bone Mineral Density; BMI: Body Mass Index; CG: Control Group; DRI: Daily Recommended Intake; DXA: Dual X-Ray Absorptiometry; FFM: FatFree Mass; FM: Fat Mass; HRmax: Maximum Heart Rate; IG: Intervention Group; IRB: Institutional Review Board; LBM: Lean Body Mass; NDSR: Nutrition Data System for Research; PP: Postpartum; RDA: Recommended Dietary Allowance; RPE: Rating of Perceived Exertion; SPSS: Statistical Package for the Social Science Software; IC: Women, Infants, and Children Program; YWCA: Young Women's Christian Association

\section{Introduction}

Female overweight and obesity rates continue to increase, and the childbearing years may increase the risk because of excessive gestational weight gain and postpartum (PP) weight retention seen in many women [1-3]. Overweight and obesity (defined as a body mass index or BMI $\geq 25.0 \mathrm{~kg} / \mathrm{m}^{2}$ ) affect nearly 70 percent of women in the United States [4]. There is a need for prevention of excessive gestational weight gain and effective treatment for women who gain excessive amounts of weight during pregnancy [5] and retain that excess weight after pregnancy. Breastfeeding may be one effective treatment to reduce excessive PP weight retention due to its high energy requirements that utilize approximately 500 calories daily [6]. 
Conversely, breastfeeding marks a time of change in maternal bone mineral density (BMD) due to the transfer of calcium in breast milk to the infant, potentially increasing the maternal risk of osteoporosis later in life. This decrease in BMD may also result from insufficient dietary calcium intake as breastfeeding amount and duration alters calcium recommended dietary allowance (RDA) [7]. Nutritional interventions that included increased dairy consumption were investigated in pre-menopausal women on body composition. Few studies have shown no significant effect of increased dairy consumption on BMD. However, no studies to date have examined breastfeeding/lactating women's dairy consumption effect on BMD loss. Additionally, exercise, particularly aerobic and resistance exercise, has shown positive effects on body composition in breastfeeding/lactating women's body composition and may slow BMD loss $[8,9]$.

Although lactation-induced bone loss is usually recovered upon weaning, not all women return to their pre-pregnancy BMD. In order to improve breastfeeding/ lactating women's body composition, new approaches are needed to reduce BMD loss, improve dietary calcium intake, increase fat-free mass (FFM) and promote fat mass (FM) loss. Dairy or yogurt consumption along with weightbearing and aerobic exercise, has shown to be a healthy approach to improving long term outcomes in breastfeeding women.

\section{Objective of the Study}

The objective of this study was to investigate the influence of yogurt supplementation and exercise on the attenuation of lactation-induced bone loss from 8-weeks to 20-weeks postpartum (PP).

\section{Materials and Methods}

Participants were recruited in the Guilford County area hospitals, pregnancy clinics, breastfeeding support groups, libraries, prenatal yoga classes, obstetricians' offices, and social media. Flyers were distributed in these locations to attract interested participants to contact research staff via email or telephone. Research staff used an initial screening form to determine eligibility during pregnancy to gather potentially interested participants. Two weeks past the reported due date, a follow-up call was made to assess interest and postnatal eligibility. Inclusion criteria were 25 to 40 years of age, fully lactating women, full-term pregnancy (> 36 weeks), cesarean or vaginal singleton birth, self-reported BMI between $22-35 \mathrm{~kg} / \mathrm{m}^{2}$ and sedentary for the past three months. The exclusion criteria were diagnosis of a medical condition that affected hormones or exercise-contradicted medical complications, women having greater than a singleton birth, smokers, and formula feeding of infants ( $>4$ oz given on occasion).

A randomized intervention study was used. Participation in the study was voluntary, and all eligible participants signed a written informed consent and agreed to randomization before any measurements occurred. Confidentiality and privacy were upheld by de-identifying participants' names and keeping all information in a secure database and locked storage cabinet in the research laboratory. Biological samples collected were also de-identified and stored appropriately. The Institutional Review Board (IRB) at North Carolina Agricultural and Technical State University approved this study (IRB\#: 18-0010). This study was registered with ClinicalTrials.gov (NCT03732261). Due to COVID-19 the study was prematurely ended in March 2020.

\section{Laboratory measurements}

All eligible participants participated in measurements at baseline ( $7 \pm 2$ wk PP) and endpoint (19 \pm 2 wk PP). Laboratory measurements included demographics, maternal and infant anthropometrics, DXA, cardiovascular treadmill testing, and dietary intake.

Height and weight to obtain body mass index (BMI) were measured for the mothers using a digital scale and standardized stadiometer (Health 0 Meter 500KL digital physicians scale). All particiants were instructed to wear light clothing and remove shoes. Infant weight and length were measured using a RICE Lake infanct scale and Seca Model 416 length board, respectively. A Gulick tape measure was used to measure waist circumference, the narrowest point above the umbilicus and below the xiphoid process, and hip circumference. All measurements were repeated twice to ensure accuracy; the average of the two measurements was taken as the final recorded measurement. Body composition was measured using DXA (Lunar Prodigy Advance from GE Medical Systems). DXA is a three-compartment model non-invasive test to measure body composition (FM and FFM) and bone density with one test. The scan focuses on two sites, lumbar spine and total neck (trochanter, Wards, femoral neck), and is important to assess an individual's risk of osteoporosis.

\section{Assessment of cardiovascular fitness}

A treadmill test was administered to measure participants' cardiovascular fitness. Resting blood pressure and heart rate was obtained using a sphygmomanometer. Once heart rate was mea- 
sured, target heart rate was calculated to find $80 \%$ of participant's maximum heart rate (HRmax). Participants' heart rate and Rating of Perceived Exertion (RPE) were monitored during the test using the RPE scale and heart rate monitor until $80 \%$ of their maximum heart rate was achieved. During the test, the grade of the treadmill increased by 2.5 after the completion of each stage, and the participant was asked to report a number on the RPE scale every minute. Once HRmax was reached, the test stopped, and the cool down phase began to get the participant's heart rate below $120 \mathrm{bpm}$.

Assessment of dietary intake

Dietary intake was assessed by 24-hour dietary recalls completed for 3 days using the Nutrition Data System for Research (NDSR 2019, University of Minnesota) via telephone or in-person interviews during baseline and endpoint measurements. This method is accurate and has been validated in previous research studies against doubly-labeled water for assessing dietary intake in groups $[10,11]$. This system utilizes a 24-hour dietary recall automated multiple-pass method. A single 24-hour recall is an inexpensive way to record detailed information for all intake in the past recent 24-hours. However, many individuals tend to either underreport or overreport as it relies on the participant's memory. A food amounts booklet was given to the participants, and they were instructed to use the booklet during each 24-hour dietary recall interview to ensure the accuracy of measurement for reporting in the system.

\section{Study intervention}

\section{Intervention group}

The intervention group (IG) for this study participated in a three-day per week 12-week exercise and dietary intervention. Women randomized to the IG were given a year membership to a community-based exercise program (North Gateway Research Center, Greensboro, NC), a pedometer, and individualized dietary recommendations. Community-based group exercise sessions led by trained research assistants ranged from 45 minutes to 60 minutes in duration. Exercises were progressive, targeting the core and individualized based on each participant's physical strength. The first four weeks of the program focused on form and foundational exercises (e.g. squats, bench, rows, deadlifts and military press). The second four weeks progressed into combination exercises and gentle jumping (e.g. jumping jacks, squat jumps). The exercises continued to focus on engaging the core and stressing bone, particularly the lumbar spine, in multiplanar directions while progressing the exercise program. The final four weeks increased the intensity through H.I.I.T. (high intensity interval training) building on the foundational exercises and progression through combination exercises (e.g. burpees squats, pushups, overhead press). Additionally, participants were encouraged to walk 10,000 steps per day. Childcare and breastfeeding support were provided to the women at each exercise session. The weekly workout (sets, reps and weight - body weight, 8 pound or 10 pound dumbbells) and steps were recorded by research assistants. Participants also reported any other daily exercise activities they did outside the study's exercise sessions during the week.

For the dietary intervention, the women were provided daily six-ounces of either plain or vanilla yogurt fortified with vitamin D for the 12-week intervention. The nutritional content of the yogurt provided $250 \mathrm{mg}$ of calcium (25\% DRI, $1000 \mathrm{mg}$ [5]), 8g of protein, and less than 110 calories. Participants self-reported any additional daily dairy intake, and this was recorded weekly by the research assistants. Weekly one-on-one nutrition education was explained to each participant by a Registered Dietitian and research assistants after each workout session. Every week focused on different topics geared towards lactating women and children.

\section{Control group}

Women randomized into the control group (CG) in this study were asked not to participate in any structured exercise or make any changes to their usual diet. The participants were allowed to walk their infants in strollers at a leisurely pace (no faster than two mph) for no more than 30 minutes per day during the 12-week study period. After the endpoint measurements (18 - 20 weeks PP) of the 12week intervention, the CG was asked to join the community-based exercise program provided to the IG. The participants were given the same materials given to the IG such as a pedometer, year membership to the North Gateway Research Campus exercise facility, individual dietary recommendations, and nutrition education handouts. Additionally, support by the PI and research assistants for exercise and diet were offered until the one-year PP laboratory measurement.

\section{Statistical analysis}

The data collected was analyzed using Statistical Package for the Social Science software (SPSS) (IBM Corp. Released 2017. IBM SPSS Statistics for Windows, Version 23.0. Armonk, NY: IBM Corp). The demographic data were reported using descriptive statistics (mean, standard deviation, and percentage). A paired t-test was used to compare the differences between the mean of baseline 
and endpoint measurements in the intervention group for body composition, BMD, and cardiovascular fitness. Due to COVID-19, statistical analysis to compare the intervention group with control group $(\mathrm{n}=1)$ was not performed on all measures except change in dietary intake. A two-way ANOVA was used to test significance of baseline characteristics between groups. Repeated measures ANOVA was used to compare means of IG to CG for dietary intake only. Results are reported as mean $\pm \mathrm{SD}$, with statistical significance set at $\mathrm{p}<0.05$.

\section{Results}

From April 2019 to March 2020, research assistants recruited at over 70 in-person community events targeted towards pregnant, PP women, and children. Recruitment was conducted mainly in the Guilford County area. Sites included WIC-Greensboro and High Point, La Leche League, YWCA, and Mahogany Milk. Twenty-nine women were screened after expressing initial interest in the study to determine eligibility; however, thirteen women were deemed ineligible after initial screening or after delivery. Eight women completed baseline measurements (Figure 1). The IG (n =6) came in for baseline measurements between 6 - 8 weeks PP; however, one woman completed her baseline measurements at 5 weeks PP because she was not available during the 6 - 8-week time period. The CG randomized two participants. Baseline and endpoint measurements were completed on five of the participants at $6.4 \pm 1.5$ weeks PP and $20.2 \pm 0.8$ weeks PP, respectively. Due to COVID-19 and the statewide stay-at-home order, three of the participants have all baseline data with dietary intake only for endpoint data. All participants had a college degree or higher and in a higher socioeconomic status. The study had a diverse sample: two Asians, two African Americans, and four Caucasians. Six participants were stay-at-home moms or had flexible work-from-home jobs. Two worked full time out of their home.

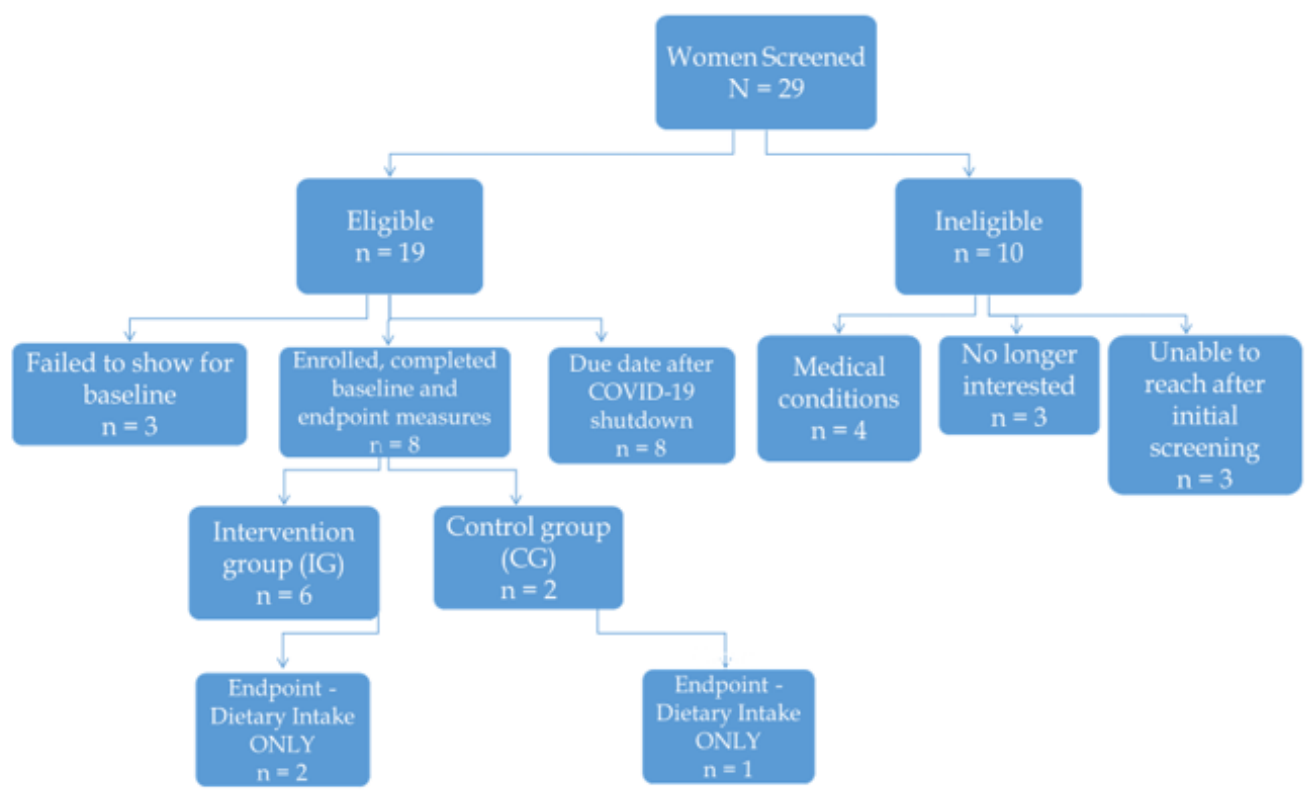

Figure 1: MEEMA Study (IG n =6, CG n = 2).

There were no significant differences between groups for any baseline anthropometric measurements, $p>0.1$ (Table 1). The IG lost $3.4 \%$ of their body weight $(n=4)$, however, the change was not significant ( $p>0.1$ ) (Table 2). The IG lost BMD at all sites with the greatest loss at the lumbar spine and total hip, $1.4 \% \pm 2.1 \%$ and $-1.4 \% \pm 2.0 \%$, respectively (Table 3 ). The IG loss in total body BMD was significant, $\mathrm{p}=0.008$.
IG group $(\mathrm{n}=4)$ increased their $\mathrm{VO}_{2} \max 8 \% \pm 7 \%$; however the IG change was not statistically significant $(p=0.09)$. Compliance for IG exercise three days per week was $90 \% \pm 12 \%$. Women in the IG were expected to reach 10,000 aerobic steps per day. Participants failed to meet the 10,000 goal, with a daily average of 5400 steps per day. 


\begin{tabular}{|c|c|c|c|c|}
\hline \multirow[t]{2}{*}{ Characteristic } & \multicolumn{2}{|c|}{ Intervention group $(n=6)$} & \multicolumn{2}{|c|}{ Control group $(n=2)$} \\
\hline & Mean (SD) & Range & Mean (SD) & Range \\
\hline Age (year) & $35.2(3.1)$ & $31-39$ & $31.0(1.4)$ & $30-32$ \\
\hline Pre-pregnancy weight (kg) & $68.0(11.2)$ & $56.0-62.2$ & $68.8(24.3)$ & $55.1-60.9$ \\
\hline \multicolumn{5}{|l|}{ Weight of infants (kg) } \\
\hline Birth & $3.7(0.4)$ & $3.3-4.3$ & $3.7(0.2)$ & $3.5-3.9$ \\
\hline Baseline & $5.5(1.0)$ & $4.5-7.0$ & $5.9(0.8)$ & $5.3-6.5$ \\
\hline Gain (kg, 6.4 to 20.2 weeks) & $1.9(0.2)^{2}$ & $1.5-2.0^{2}$ & $1.5(0.0)^{2}$ & $\cdots$ \\
\hline \multicolumn{5}{|l|}{ Sex of infant (no.) } \\
\hline Female & \multicolumn{2}{|c|}{2} & \multicolumn{2}{|c|}{1} \\
\hline Male & \multicolumn{2}{|c|}{4} & \multicolumn{2}{|c|}{1} \\
\hline \multicolumn{5}{|l|}{ Parity } \\
\hline Primiparous & \multicolumn{2}{|c|}{1} & \multicolumn{2}{|c|}{0} \\
\hline Mutliparous & \multicolumn{2}{|c|}{5} & \multicolumn{2}{|c|}{2} \\
\hline
\end{tabular}

Table 1: Baseline characteristics of the intervention and control group ${ }^{1}$.

1: Data are mean (SD), $\mathrm{p}>0.05$.

2: Due to COVID-19, endpoint measurements were obtained for $n=4$ IG; $n=1$ CG.

\begin{tabular}{|c|c|c|c|c|}
\hline & \multicolumn{2}{|c|}{ Intervention Group } & \multicolumn{2}{|c|}{ Control Group } \\
\hline & Baseline $(n=6)$ & Endpoint $(n=4)^{2}$ & Baseline $(n=2)$ & Endpoint $(n=1)^{2}$ \\
\hline & \multicolumn{4}{|c|}{-Mean (SD) - } \\
\hline Weight (kg) & $73.6(9.3)$ & $71.2(14.9)$ & $73.8(24.8)$ & $93.6(0.0)$ \\
\hline & $60.8-83.4$ & $56.9-86.3$ & $56.2-91.3$ & - \\
\hline BMI $\left(\mathrm{kg} / \mathrm{m}^{2}\right)$ & $27.7(2.6)$ & $26.8(3.4)$ & $29.8(11.5)$ & $38.3(0.0)$ \\
\hline & $24.2-30.5$ & $23.3-30.7$ & $37.9-21.6$ & - \\
\hline \multicolumn{5}{|l|}{ Body fat } \\
\hline \multirow[t]{2}{*}{$\mathrm{Kg}$} & $30.2(4.1)$ & $27.4(7.3)$ & $30.8(22.2)$ & $44.1(0.0)$ \\
\hline & $23.7-35.0$ & 20.1-36.4 & $15.1-46.5$ & - \\
\hline \multirow[t]{2}{*}{ Percent } & $42.2(2.2)$ & $39.7(3.1)$ & $41.0(17.9)$ & $49.4(0.0)$ \\
\hline & 39.1-44.9 & $36.8-44.0$ & $28.3-53.6$ & - \\
\hline \multirow[t]{2}{*}{ LBM $(\mathrm{kg})$} & $41.4(5.7)$ & $53.6(22.1)$ & $39.3(1.4)$ & $45.2(0.0)$ \\
\hline & $34.3-49.0$ & $34.5-85.5$ & 40.3-38.3 & - \\
\hline \multirow[t]{2}{*}{ Fat-free mass } & $44.0(6.0)$ & $43.6(7.6)$ & $42.2(2.3)$ & $48.3(0.0)$ \\
\hline & $36.5-51.8$ & $36.6-51.0$ & $40.6-43.8$ & - \\
\hline \multirow[t]{2}{*}{ Waist circumference $(\mathrm{cm})$} & $85.8(5.1)$ & $83.2(8.3)$ & $84.8(13.4)$ & $93.5(0.0)$ \\
\hline & $80.5-92.9$ & $74.7-92.7$ & $75.3-94.3$ & - \\
\hline \multirow[t]{2}{*}{ Hip circumference $(\mathrm{cm})$} & $106.9(6.4)$ & $104.3(9.4)$ & $110.9(17.0)$ & $124.9(0.0)$ \\
\hline & 100.9-117.2 & $95.2-116.3$ & $75.3-94.3$ & - \\
\hline
\end{tabular}

Table 2: Body composition of the intervention and control groups ${ }^{1}$.

1: LBM = lean body mass (non-bone); Fat Free Mass = LBM + Bone Mineral Content (BMC) Mean (SD)

2: Due to COVID-19 endpoint measurements were obtained for $\mathrm{n}=4 \mathrm{IG} ; \mathrm{n}=1 \mathrm{CG}$. 


\begin{tabular}{|c|c|c|c|c|}
\hline & \multicolumn{2}{|c|}{ Intervention Group } & \multicolumn{2}{|c|}{ Control Group } \\
\hline & Baseline $(n=6)$ & Endpoint $(n=4)^{2}$ & Baseline $(n=2)$ & Endpoint $(n=1)^{2}$ \\
\hline & \multicolumn{4}{|c|}{ - } \\
\hline \multicolumn{5}{|l|}{ Total body } \\
\hline \multirow[t]{2}{*}{$\operatorname{BMD}\left(\mathrm{g} / \mathrm{cm}^{2}\right)$} & $1.178(0.068)$ & $1.161(0.088)^{\mathrm{a}}$ & $1.244(0.137)$ & $1.317(0.0)$ \\
\hline & $1.092-1.260$ & $1.083-1.256$ & $1.147-1.341$ & - \\
\hline \multirow[t]{2}{*}{$\mathrm{BMC}(\mathrm{g})$} & $2564(343)$ & 2444 (417) & 2827 (832) & $3122(0.0)$ \\
\hline & $2127-2893$ & 2061-2829 & $2238-3415$ & - \\
\hline \multirow[t]{2}{*}{ Area $\left(\mathrm{cm}^{2}\right)$} & $2169(186)$ & 2095 (204) & $2250(421)$ & $2371(0.0)$ \\
\hline & $2169-2403$ & $1894-2287$ & $1952-2547$ & - \\
\hline \multicolumn{5}{|l|}{ Lumbar spine } \\
\hline \multirow[t]{2}{*}{$\mathrm{BMD}\left(\mathrm{g} / \mathrm{cm}^{2}\right)$} & $1.160(0.084)$ & $1.186(0.061)$ & $1.197(0.223)$ & $1.365(0.0)$ \\
\hline & $1.283-1.1595$ & $1.133-1.268$ & $1.039-1.354$ & - \\
\hline \multirow[t]{2}{*}{$\mathrm{BMC}(\mathrm{g})$} & $59.6(7.5)$ & $59.8(7.1)$ & $60.1(12.9)$ & $68.9(0.0)$ \\
\hline & $53.0-73.7$ & $52.1-69.3$ & $51.0-69.2$ & - \\
\hline \multirow[t]{2}{*}{ Area $\left(\mathrm{cm}^{2}\right)$} & $51.3(3.8)$ & $50.3(3.8)$ & $50.1(1.4)$ & $50.5(0.0)$ \\
\hline & $46.1-57.4$ & $46.0-54.7$ & 49.1-51.1 & - \\
\hline \multicolumn{5}{|l|}{ Total hip } \\
\hline \multirow[t]{2}{*}{$\operatorname{BMD}\left(\mathrm{g} / \mathrm{cm}^{2}\right)$} & $0.989(0.127)$ & $1.013(0.127)$ & $1.139(0.221)$ & $1.235(0.0)$ \\
\hline & $0.840-1.184$ & $0.905-1.172$ & $0.983-1.295$ & - \\
\hline \multirow[t]{2}{*}{$\mathrm{BMC}(\mathrm{g})$} & $29.8(5.7)$ & $30.3(5.6)$ & $33.3(7.8)$ & $36.5(0.0)$ \\
\hline & $23.2-36.7$ & $25.3-35.9$ & $38.8-27.7$ & - \\
\hline \multirow[t]{2}{*}{ Area $\left(\mathrm{cm}^{2}\right)$} & $29.9(2.4)$ & $29.8(2.2)$ & $29.1(1.2)$ & $29.5(0.0)$ \\
\hline & $27.6-32.7$ & $28.0-32.5$ & $30.0-28.2$ & - \\
\hline \multicolumn{5}{|l|}{ Trochanter } \\
\hline \multirow[t]{2}{*}{$\operatorname{BMD}\left(\mathrm{g} / \mathrm{cm}^{2}\right)$} & $0.774(0.118)$ & $0.791(0.091)$ & $0.888(0.248)$ & $0.993(0.0)$ \\
\hline & 0.594-0.912 & $0.700-0.904$ & $0.712-1.063$ & - \\
\hline \multirow[t]{2}{*}{$\mathrm{BMC}(\mathrm{g})$} & $9.5(2.6)$ & $9.5(1.9)$ & $10.8(2.8)$ & $11.7(0.0)$ \\
\hline & $6.0-12.1$ & $7.7-11.2$ & 8.8-12.7 & - \\
\hline \multirow[t]{2}{*}{ Area $\left(\mathrm{cm}^{2}\right)$} & $12.0(1.7)$ & $12.0(1.3)$ & $12.2(0.2)$ & $11.7(0.0)$ \\
\hline & $10.0-14.0$ & $11.0-13.7$ & $12.0-12.3$ & - \\
\hline
\end{tabular}

Table 3: Bone mineral density of the intervention and control groups.

1: Data are mean (SD).

2: Due to COVID-19 endpoint measurements were obtained for $n=4$ IG; $n=1$ CG.

a: Paired t-test IG only, change from baseline to endpoint, $\mathrm{p}=0.008$. 
There were no significant dietary differences observed between groups, except dietary calcium intake (Table 4). Both groups met the Recommended Dietary Allowance for carbohydrates and protein (g) intake; however, the CG was below recommendations for protein when body weight was considered. Both groups failed to meet the RDA for dietary Vitamin D; however, when prenatal supplements are accounted for, both groups met the RDA.
Compliance for IG for three days per week of yogurt consumption was $97 \% \pm 6 \%$. On average, the IG consumed $9.6 \pm 3.8$ servings of dairy per week (1.4 servings per day). The dairy recommendations for PP lactating women are 2 - 3 servings per day, therefore the group did not meet the weekly (daily) nutrition recommendations. However, one participant failed to report their dairy intake over the course of the 12-week intervention and were excluded from the analysis.

\begin{tabular}{|c|c|c|c|c|c|}
\hline & \multicolumn{2}{|c|}{ Intervention Group $(n=6)$} & \multicolumn{2}{|c|}{ Control Group $(n=2)$} & \multirow[t]{2}{*}{ RDA*/AI+ [5] } \\
\hline & Baseline & Endpoint & Baseline & Endpoint & \\
\hline & \multicolumn{5}{|c|}{ - } \\
\hline \multicolumn{6}{|l|}{ Energy } \\
\hline \multirow[t]{2}{*}{ kcal } & 2255 (459) & $2465(541)$ & $2038(956)$ & 1875 (205) & $+300 \mathrm{kcal}^{*}$ \\
\hline & $1665-2682$ & 1901-3293 & $1362-2714$ & $1730-2020$ & \\
\hline \multirow[t]{2}{*}{$\mathrm{kcal} / \mathrm{kg}$ body weight } & $30.9(6.1)$ & $33.1(11.9)^{2}$ & $31.6(23.6)$ & $21.6(0.0)^{2}$ & \\
\hline & $20-37$ & $22-50$ & $15-48$ & - & \\
\hline \multirow[t]{2}{*}{ Carbohydrate (g) } & $218(31)$ & $257(51)$ & $286(175)$ & $238(1)$ & $210 \mathrm{~g} / \mathrm{d}^{*}$ \\
\hline & $189-264$ & $203-346$ & $163-410$ & $237-239$ & \\
\hline \multirow[t]{2}{*}{ Protein (g) } & $92(20)$ & $94(22)$ & $80(2)$ & $71(5)$ & $71 \mathrm{~g} / \mathrm{d}^{*}$ \\
\hline & $61-117$ & $75-1129$ & $79-81$ & $67-74$ & \\
\hline \multirow[t]{2}{*}{ Protein ( $\mathrm{g} / \mathrm{kg}$ body weight) } & $1.3(0.3)$ & $1.4(0.6)^{2}$ & $1.2(0.4)$ & $0.7(0.0)^{2}$ & $1.3 \mathrm{~g} / \mathrm{kg} / \mathrm{d}^{*}$ \\
\hline & $0.7-1.6$ & $1.0-2.3$ & $0.9-1.4$ & - & \\
\hline \multirow[t]{2}{*}{ Fat $(g)$} & $110(37)$ & $110(32)$ & $80(14)$ & $73(23)$ & \\
\hline & $57-145$ & $71-156$ & $71-90$ & $57-89$ & \\
\hline \multirow[t]{2}{*}{ Saturated fat (g) } & $36(13)$ & $39(11)$ & $27(7)$ & $21(12)$ & \\
\hline & $21-49$ & $28-54$ & $22-32$ & $12-30$ & \\
\hline \multirow[t]{2}{*}{ Fiber $(\mathrm{g})$} & $22(6)$ & $25(8)$ & $19(13)$ & $14(2)$ & $29 \mathrm{~g} / \mathrm{d}^{+}$ \\
\hline & $13-31$ & $14-37$ & $10-27$ & $12-15$ & \\
\hline \multirow[t]{2}{*}{ Calcium (mg) } & 864 (274) & 977 (155) & 816 (55) & $588(85)$ & $1000 \mathrm{~g} / \mathrm{d}^{*}$ \\
\hline & $551-1188$ & $822-1228$ & $777-855$ & $528-942$ & \\
\hline \multirow[t]{2}{*}{ Phosphorus (mg) } & $1433(332)$ & 1411 (399) & $1270(354)$ & 914 (39) & $700 \mathrm{mg} / \mathrm{d}^{*}$ \\
\hline & $992-1821$ & $954-2077$ & $1019-1520$ & $887-942$ & \\
\hline \multirow[t]{2}{*}{ Magnesium (mg) } & $344(86)$ & $356(122)$ & 299 (169) & 226 (9) & $320 \mathrm{mg} / \mathrm{d}^{*}$ \\
\hline & $228-444$ & $223-546$ & $179-418$ & $219-232$ & \\
\hline \multirow[t]{2}{*}{ Vitamin D (mcg) } & $10(9)$ & $2(1)$ & $8(8)$ & $4(3)$ & $15 \mathrm{mcg} / \mathrm{d}^{*}$ \\
\hline & $2-25$ & $1-3$ & $3-14$ & $2-7$ & \\
\hline
\end{tabular}

Table 4: Dietary intake of the intervention and control groups ${ }^{1}$.

${ }^{1}$ : Data are mean (SD), no difference between groups or over time $\mathrm{p} \geq 0.05$

2: Due to COVID-19 endpoint measurements were obtained for $\mathrm{n}=4$ IG; $\mathrm{n}=1$ CG. Missing endpoint body weight measurement ( $\mathrm{n}=1)$.

*: Recommended Dietary Allowance (RDA) first six months of lactation (macronutrients); lactation ages 31 - 50.

${ }^{+}$: Adequate Intakes (AI) first six months of lactation (macronutrients); lactation ages 31 - 50. 


\section{Discussion}

The purpose of this study was to determine whether a combination of yogurt supplementation and weight-bearing aerobic exercise attenuated bone loss from 8 to 20 weeks PP in lactating women. The study design was modeled after two previous studies $[8,9]$. Unfortunately, due to COVID-19, the study was ended prematurely with three participants at different time points in the 12 -week intervention. The two participants in the intervention group were able to successfully complete the exercise intervention at home. However, weekly yogurt delivery from the study had to stop for the intervention group, and only endpoint dietary data was able to be collected from both groups. Despite the prematurely ending of the study, the four participants in the IG who completed all endpoint measurements lost $3.4 \%$ of their body weight ( $3 \mathrm{~kg}$ ), equivalent to a half a pound per week weight loss. This result was similar to weight average weight loss seen in the two previous studies $[8,9]$. Additionally, the weight loss from this study intervention was from fat-mass. The IG $(n=4)$ reduced their body fat percentage by $5 \%$ and increase LBM by $29 \%$, on average. Cardiorespiratory fitness $\left(\mathrm{VO}_{2} \mathrm{max}\right)$ also increased in these four women by a modest $8 \%$. Our findings for the IG were similar to previous studies that observed the effects of dairy (fermented and non-fermented) supplementation in reduction of body fat in pre- and post-menopausal women and found fermented-dairy intake resulted in increased body fat loss [12-15]. High protein fermented dairy or yogurt supplementation after performing moderately intense weight-bearing and aerobic exercise may reduce body fat and increase LBM. Increased physical activity is most likely the predictor for increased cardiovascular fitness seen in the IG.

Similar to previous studies [8,9], IG $(n=4)$ loss $0.9 \%$ total body BMD. However, the loss of BMD in the lumbar spine and hip were less. Additionally, IG lost less total hip BMC which was a trend reported in previous studies $[8,16,17]$. These findings still support previous studies that observed decreased femoral neck BMD loss in women who participated in weight-bearing and/or aerobic exercise interventions $[8,9]$.

The dietary intake of both groups was not significantly different. However, the IG $(n=6)$ maintained their energy (kcal) intake during the intervention and improved macro- and micronutrient intake, except for Vitamin D. Dietary Vitamin D intake failed to meet the RDA (15 mcg/d). All women in the study maintained a daily intake of prenatal vitamins which contain on average $10 \mathrm{mcg}$ (400 IU). The weekly nutrition education provided during workouts help the IG improved their dietary intake throughout the study. This finding implies that participants' nutritional awareness on adequate intake of specific nutrients during the lactation time period was improved in the IG and this did translate into the appropriate dietary intake.

Previous studies that have utilized nutrition interventions aimed at lactating women have also reported the IGs meeting or exceeding RDAs for select nutrients, specifically calcium $[8,9,18]$. In one study comparing premenopausal women consuming a low -calcium versus high-calcium diet, increased BMD was observed in those with a high-calcium diet that exceeded dietary calcium recommendations versus BMD loss in those with low-calcium diets. Although those on the low-calcium diet had greater LBM and fat loss, those on the high-calcium diet that exceeded dietary calcium experienced a slower rate of fat loss [19].

This study did have some limitations. Due to COVID-19, recruitment efforts and the study intervention were discontinued three women were unable to complete all endpoint measurements, yielding a small sample size. A small sample size made it difficult to generalize these findings for a specific population. Randomization was the second limitation due to only two participants being randomized into the CG. The final limitation of this study was possible underreporting or overreporting of dietary intake on three-day recalls. A major difference was seen in energy consumption in the CG when comparing baseline to endpoint measurements. Under- or over-reporting may cause significant changes in dietary intake to go unobserved. Additionally, the two women in the control group BMI ranged from $21.6 \mathrm{~kg} / \mathrm{m}^{2}$ to $37.9 \mathrm{~kg} / \mathrm{m}^{2}$ giving a wide spectrum of body composition and dietary intake needs, as well as bone mineral density.

\section{Conclusion}

In summary, this study provided preliminary data on the effects of yogurt supplementation and weight-bearing strength and aerobic exercise may decrease body fat and attenuate bone loss in the lumbar spine and hip, specifically the trochanter during lactation. Additionally, weekly nutrition education increased nutritional awareness on the importance of nutrition for bone and maternal health during lactation. 
Lactation-induced bone loss is still elusive and warrants additional long-term preventative research. Future research should focus on the long- term changes on BMD, area, geometry, and content in women who exercise and have breastfed for different durations compared to women who did not initiate breastfeeding at all.

Additionally, the effects of fermented dairy or yogurt supplementation on body composition should be further investigated to determine if the high protein content combined with dietary calcium and vitamin D increase body fat loss and minimize bone turnover seen in the trabecular regions of lactating women. Lastly, nutrition interventions aimed at PP lactating women should focus on general food safety and nutrition knowledge while breastfeeding.

\section{Acknowledgements}

This publication was made possible by grant number NC.X.309.5-18-170-1 from the National Institute of Food and Agriculture (NIFA) and the Department of Family and Consumer Sciences and the Agriculture Research Station at North Carolina Agriculture and Technical State University (Greensboro, NC, USA).

\section{Conflict of Interest}

The authors declare no conflict of interest.

\section{Bibliography}

1. Amorim Adegboye AR and YM Linne. "Diet or Exercise, or Both, for Weight Reduction in Women after Childbirth". The Cochrane Database of Systematic Reviews 7 (2013): Cd005627.

2. Lim S., et al. "Effective Strategies for Weight Loss in Post-Partum Women: A Systematic Review and Meta-Analysis". Obesity Review 16.11 (2015): 972-987.

3. Lovelady CA. "Balancing Exercise and Food Intake with Lactation to Promote Post-Partum Weight Loss". Proceedings of the Nutrition Society 70.2 (2011): 181-184.

4. National Institutes of Health. "Pregnancy, Breastfeeding and Bone Health" (2015).

5. Butte NF. "Dieting and Exercise in Overweight, Lactating Women". The New England Journal of Medicine 342.7 (2000): 502-503.

6. Institute of Medicine (US) Committee on Nutritional Status During Pregnancy and Lactation. Nutrition During Lactation. Meeting Maternal Nutrient Needs During Lactation. Washington, DC 5 (1991).
7. National Institutes of Health. "Pregnancy, Breastfeeding and Bone Health" (2018).

8. Colleran HL., et al. "Effects of Energy Restriction and Exercise on Bone Mineral Density During Lactation". Medicine and Science in Sports and Exercise 44.8 (2012): 1570-1579.

9. Lovelady CA., et al. "Effect of Exercise Training on Loss of Bone Mineral Density During Lactation". Medicine and Science in Sports and Exercise 41.10 (2009): 1902-1907.

10. Blanton CA., et al. "The Usda Automated Multiple-Pass Method Accurately Estimates Group Total Energy and Nutrient Intake". Journal of Nutrition 136.10 (2006): 2594-2599.

11. Moshfegh AJ., et al. "The Us Department of Agriculture Automated Multiple-Pass Method Reduces Bias in the Collection of Energy Intakes". The American Journal of Clinical Nutrition 88.2 (2008): 324-332.

12. Josse AR., et al. "Body Composition and Strength Changes in Women with Milk and Resistance Exercise". Medicine and Science in Sports and Exercise 42.6 (2010): 1122-1130.

13. Michaelsson K., et al. "Intake of Milk or Fermented Milk Combined with Fruit and Vegetable Consumption in Relation to Hip Fracture Rates: A Cohort Study of Swedish Women". Journal of Bone and Mineral Research 33.3 (2018): 449-457.

14. White KM., et al. "Changes in Body Composition with Yogurt Consumption During Resistance Training in Women". International Journal of Sport Nutrition and Exercise Metabolism 19.1 (2009): 18-33.

15. Zemel MB., et al. "Dairy Augmentation of Total and Central Fat Loss in Obese Subjects". International Journal of Obesity (London) 29.4 (2005): 391-397.

16. Colleran HL., et al. "The Effect of an Exercise Intervention During Early Lactation on Bone Mineral Density During the First Year Postpartum". Journal of Physical Activity and Health 16.3 (2019): 197-204.

17. Soltani S., et al. "The Effects of Weight Loss Approaches on Bone Mineral Density in Adults: A Systematic Review and Meta-Analysis of Randomized Controlled Trials". Osteoporosis International 27.9 (2016): 2655-2671.

18. Krebs Nancy F., et al. "Bone Mineral Density Changes During Lactation: Maternal, Dietary, and Biochemical Correlates". The American Journal of Clinical Nutrition 65.6 (1997): 1738-1746. 
19. Thomas DT., et al. "Effects of Calcium and Resistance Exercise on Body Composition in Overweight Premenopausal Women". The Journal of the American College of Nutrition 29.6 (2010): 604-611.

\section{Assets from publication with us}

- Prompt Acknowledgement after receiving the article

- Thorough Double blinded peer review

- Rapid Publication

- Issue of Publication Certificate

- High visibility of your Published work

Website: www.actascientific.com/

Submit Article: www.actascientific.com/submission.php

Email us: editor@actascientific.com

Contact us: +919182824667 